\title{
Feasibility of stereotactic radiotherapy for lung lesions and conventional radiotherapy for nodal areas in primary lung malignancies
}

\author{
Yeon Joo Kim, Su Ssan Kim*, Si Yeol Song and Eun Kyung Choi
}

\begin{abstract}
Background: Combined stereotactic body radiotherapy (SBRT) for lung lesions and conventional radiotherapy (CRT) for nodal areas may be more effective than CRT alone in patients with locally advanced lung cancer.

Methods: This study included 21 patients with small primary lung tumors distant from the regional nodal areas. The SBRT dose was 40-60 Gy in 4 fractions. CRT doses were 66 Gy in 30 fractions for non-small cell lung cancer and 52.5 Gy in 25 fractions for small cell lung cancer.

Results: The median follow-up duration was 12 months, and the median survival was 13 months. The 1 year overall survival, local recurrence-free survival, and distant metastasis-free survival rates were $60.5,84.8$, and $62.1 \%$, respectively. Two patients experienced in-field local recurrence combined with out-field regional recurrence and/or distant failure. The major recurrence pattern was distant failure (crude incidence, $43 \%$ ). Three patients aged $\geq 79$ years experienced grade $\geq 3$ acute radiation pneumonitis, and one also had idiopathic interstitial pneumonia.

Conclusion: The combination of SBRT for the lung lesion and CRT for the nodal region seems to be effective and safe for lung malignancies. However, patients older in age and/or with underlying pulmonary disease require stricter lung dose constraints.
\end{abstract}

Keywords: Lung cancer, Radiotherapy, Survival, Toxicity

\section{Background}

Definitive concurrent chemoradiotherapy (CCRT) has been recommended as the standard treatment for unresectable or medically inoperable stage II-III non-small cell lung cancer (NSCLC), based on the findings of randomized prospective trials [1-3]. Definitive CCRT is also the treatment of choice for limited-stage small cell lung cancer (LS-SCLC), as indicated by two previous meta-analyses $[4,5]$. The common radiotherapy dose regimens include conventional radiotherapy (CRT) with 60-66 Gy (1.8-2 Gy/fraction once daily) for NSCLC and $45 \mathrm{~Gy}$ (1.5 Gy/fraction twice daily) or higher doses

\footnotetext{
* Correspondence: watermountain@hanmail.net

Department of Radiation Oncology, Asan Medical Center, University of Ulsan College of Medicine, 88 Olympic-ro 43-gil, Songpa-gu, Seoul 05505, South Korea
}

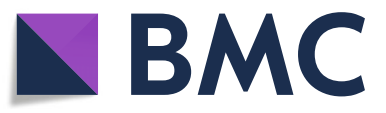

(c) The Author(s). 2018 Open Access This article is distributed under the terms of the Creative Commons Attribution 4.0 International License (http://creativecommons.org/licenses/by/4.0/), which permits unrestricted use, distribution, and reproduction in any medium, provided you give appropriate credit to the original author(s) and the source, provide a link to the Creative Commons license, and indicate if changes were made. The Creative Commons Public Domain Dedication waiver (http://creativecommons.org/publicdomain/zero/1.0/) applies to the data made available in this article, unless otherwise stated. lung cancer (SCLC).

As the primary lung lesion is located adjacent to the mediastinum and hilar lymph node (LN) region in most lung cancer patients, similar fractionation schemes are generally used for primary lung tumors and metastatic LNs. Some patients, however, are diagnosed with an isolated primary lesion, relatively small in size and distant from the nodal area (Fig. 1). Although relatively few patients present with these tumors, integrating the two separate target volumes is often difficult even when planning target volume (PTV) margins are larger. In contrast to the concept that all tumors should receive the same radiation regimens, different radiotherapy regimens could be administered to each target in these patients. 


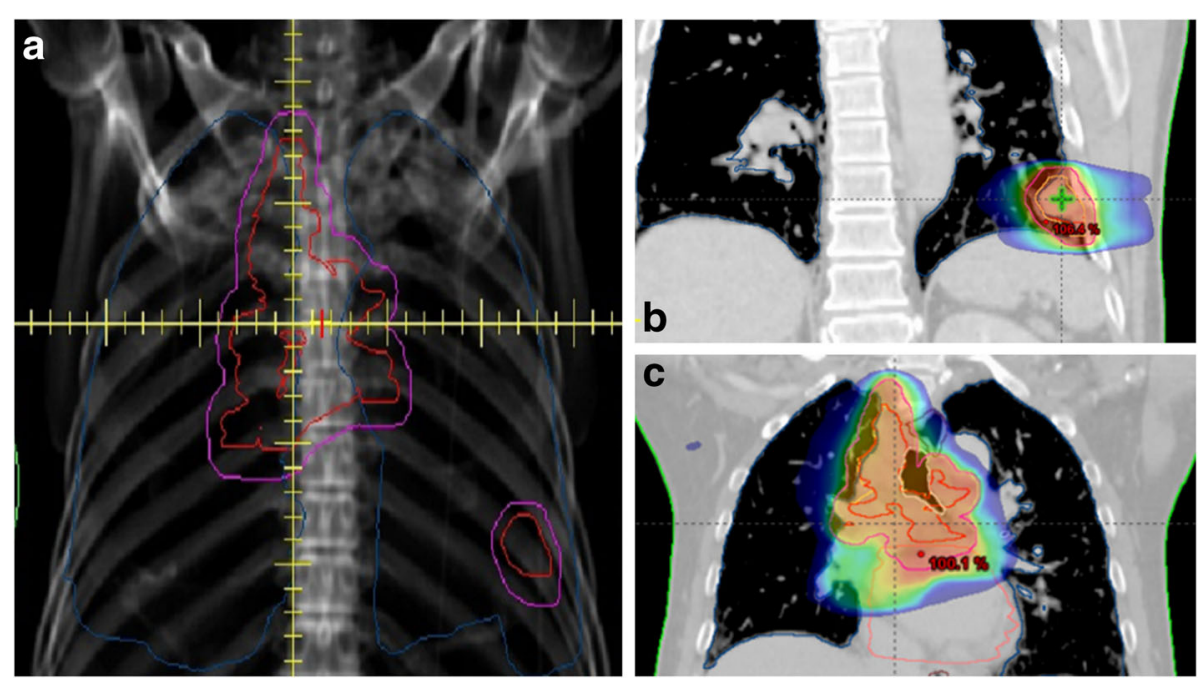

Fig. 1 Anterior digitally reconstructed radiography (a) and dose distributions in a 65-year-old man treated with stereotactic body radiotherapy for a $2.4 \mathrm{~cm}$-sized primary tumor in the left lower lobe (b), and with conventional radiotherapy for metastatic mediastinal lymph nodes (c)

Stereotactic body radiotherapy (SBRT) consists of the administration of one or a few fractions of radiation (with high doses in each fraction), and is expected to achieve improved local control. Several reports indicate the effectiveness of SBRT in the management of patients with early-stage NSCLC, with 2-3 year local control rates of approximately $90 \%$ in patients with $\mathrm{T} 1-2$ disease [6-8]. In addition, two randomized trials compared SBRT with CRT in patients with medically inoperable stage I NSCLC. The SPACE trial reported that the two regimens resulted in similar progression-free survival and overall survival (OS) rates [9], whereas the CHISEL trial found that SBRT resulted in superior local control and OS [10]. The results of the CHISEL trial suggest that administration of SBRT to the lung lesion and CRT to the nodal area could improve control of the primary lung tumor and further improve control of the entire region in advanced lung cancer patients with two distinct target volumes.

This strategy might also reduce toxicities, owing to the reduction in PTV margins and the characteristics of SBRT including high targeting accuracy and rapid dose falloff. Because the SPACE trial found that the rates of pulmonary and esophageal toxicities were lower with SBRT than with CRT [9], our center treated these patients by administering SBRT to the lung nodule and CRT to the nodal area, a regimen termed stereotactic plus conventional radiotherapy $(\mathrm{S}+\mathrm{CRT})$. This retrospective analysis assessed the efficacy and safety of S + CRT.

\section{Methods}

\section{Patients}

The records of patients diagnosed with lung malignancy and treated with $\mathrm{S}+\mathrm{CRT}$ at our hospital between March
2009 and July 2017 were retrospectively reviewed. All patients who received S + CRT had a small isolated primary lesion located distant from the nodal area (Fig. 1). Patients with distant metastases and those diagnosed with a primary cancer other than lung cancer within the previous 5 years were excluded. Patients underwent extensive examination, including pathologic confirmation, chest computed tomography (CT), 18-fluoro-deoxyglucose positron emission tomography (FDG-PET-CT), and/or brain magnetic resonance imaging. LNs of diameter $\geq$ $1 \mathrm{~cm}$ and definitive FDG uptake were classified as clinically malignant. LN metastases were pathologically confirmed whenever possible. Pulmonary function tests were performed prior to treatment. Data of all patients were reviewed, and tumor stage was determined according to the American Joint Committee on Cancer (AJCC) 7th edition TNM stage classification. SCLCs were also staged using the 7th AJCC system, based on the recommendations of the International Association for the Study of Lung Cancer [11]. The study protocol was approved by the institutional review board of our hospital, which waived the requirement for informed consent due to the retrospective nature of this study.

\section{Treatments}

For SBRT planning, four-dimensional CT images (slice thickness, $2.5 \mathrm{~mm}$ ) reflecting respiratory motion were acquired. The gross tumor volume (GTV) of the lung was delineated at the end-exhale phases using the lung setting $(\mathrm{W}=1700, \mathrm{~L}=-300)$. The internal target volume (ITV) was contoured using maximum intensity projection images, whereas the PTV was a $5 \mathrm{~mm}$ expansion of the ITV. The SBRT dose (range, 40-60 Gy) was administered in four fractions, as determined by the radiation 
oncologist. After SBRT, all patients underwent CT (slice thickness, $2.5 \mathrm{~mm}$ ) for three-dimensional CRT planning. The GTV at the LN was contoured using the mediastinal setting $(\mathrm{W}=600, \mathrm{~L}=40)$, and the PTV margins were maintained as $7 \mathrm{~mm}$ radially and $10 \mathrm{~mm}$ longitudinally. The clinical target volumes were also delineated for some patients to cover the regional nodal areas in the same axial sections; however, smaller PTV margins (5 $\mathrm{mm}$ radially and $7 \mathrm{~mm}$ longitudinally) were considered in these patients. In patients treated with induction chemotherapy, the post-chemotherapy volume was defined as the GTV. Standard radiotherapy consisted of 66 Gy (2.2 Gy/fraction) for NSCLC and 52.5 Gy (2.1 Gy/ fraction) for SCLC.

The organs at risk (OAR) included healthy lung tissues, the esophagus, the spinal cord, and the heart. The normal organ constraints included a maximal dose for the spinal cord of $<50 \mathrm{~Gy}$, a mean lung dose (MLD) of $<20 \mathrm{~Gy}$, a volume of the lung receiving at least $20 \mathrm{~Gy}$ (V20) of $<30 \%$, a mean esophagus dose of $<35$ Gy, and heart doses of $\mathrm{V} 60<1 / 3$, V $45<2 / 3$, and $\mathrm{V} 40<100 \%$.

Chest X-rays (CXR) were obtained weekly to monitor changes in tumor volume and any acute toxicities. Treatment was verified by weekly $\mathrm{kV}$ imaging guidance, using set-up correction based on carina and bony anatomy.

The main concurrent chemotherapy regimen for NSCLC consisted of weekly doses of paclitaxel combined with cisplatin or carboplatin. Patients with very large tumors and a high risk of radiation-related toxicities were initially treated with induction chemotherapy for volume reduction, with a regimen consisting of two cycles of gemcitabine plus cisplatin in sequential schedules every 3 weeks. Chemotherapy for SCLC consisted of intravenous etoposide and cisplatin administered every 3 weeks for four cycles, with radiotherapy initiated along with the third cycle of chemotherapy. Patients with a poor performance status or poor lung function were recommended to undergo radiotherapy alone.

\section{Follow-up, toxicity scoring, and statistical analysis}

All patients underwent weekly complete blood counts and CXR. Patients were routinely followed up by a radiation oncologist and/or medical oncologist, with chest CT and/or CXR performed 1 month after treatment, every 3 months during the first 2 years, and every 6 months thereafter until 5 years after treatment.

The primary outcome was 1 year OS rate. Secondary endpoints included 1 year local recurrence-free survival (LRFS) and distant metastasis-free survival (DMFS) rates and toxicities. Toxicities were evaluated using the Common Terminology Criteria for Adverse Events version 4.03. Adverse events that occurred during treatment and
Table 1 Patient and treatment characteristics

\begin{tabular}{|c|c|c|c|}
\hline \multicolumn{3}{|l|}{ Factors } & \multirow{2}{*}{$\begin{array}{l}\text { Number }(n=21) \\
17\end{array}$} \\
\hline \multirow{2}{*}{\multicolumn{2}{|c|}{ Sex }} & Male & \\
\hline & & Female & 4 \\
\hline \multicolumn{2}{|l|}{ Age (years) } & Median (range) & $68(52-88)$ \\
\hline \multirow{5}{*}{\multicolumn{2}{|c|}{ Location (lobe) ${ }^{\mathrm{a}}$}} & Right upper & 5 \\
\hline & & Right middle & 3 \\
\hline & & Right lower & 4 \\
\hline & & Left upper & 6 \\
\hline & & Left lower & 7 \\
\hline \multirow{3}{*}{\multicolumn{2}{|c|}{ T stage }} & 1 & 13 \\
\hline & & 2 & 5 \\
\hline & & 3 & 3 \\
\hline \multirow{3}{*}{\multicolumn{2}{|c|}{ N stage }} & 1 & 4 \\
\hline & & 2 & 8 \\
\hline & & 3 & 9 \\
\hline \multirow{3}{*}{\multicolumn{2}{|c|}{ Clinical stage }} & $\| \mathrm{A}$ & 4 \\
\hline & & $\| I I A$ & 8 \\
\hline & & $\| I B$ & 9 \\
\hline \multirow{3}{*}{\multicolumn{2}{|c|}{ ECOG performance status }} & 0 & 2 \\
\hline & & 1 & 16 \\
\hline & & 2 & 3 \\
\hline \multirow{2}{*}{\multicolumn{2}{|c|}{ Weight loss }} & No & 18 \\
\hline & & Yes & 3 \\
\hline \multirow{2}{*}{\multicolumn{2}{|c|}{ Smoking history }} & No & 4 \\
\hline & & Yes & 17 \\
\hline \multicolumn{2}{|l|}{ FEV1 (\%) } & Median (range) & $83(51-116)$ \\
\hline \multicolumn{2}{|l|}{ DLco $(\%)^{b}$} & Median (range) & 73 (37-99) \\
\hline \multirow{4}{*}{\multicolumn{2}{|c|}{ Pathology }} & Squamous & 8 \\
\hline & & Adenocarcinoma & 9 \\
\hline & & Unknown NSCLC & 1 \\
\hline & & $\mathrm{SCLC}$ & 3 \\
\hline \multirow{2}{*}{\multicolumn{2}{|c|}{ Aim }} & Definitive & 15 \\
\hline & & Salvage & 6 \\
\hline \multirow[t]{2}{*}{ Radiotherapy dose (Gy) } & SBRT & Median (range) & $54(40-60)$ \\
\hline & CRT & Median (range) & $60(46-66)$ \\
\hline \multirow[t]{2}{*}{ Dose per fraction (Gy) } & SBRT & Median (range) & $14(10-15)$ \\
\hline & CRT & Median (range) & $2(2-6)$ \\
\hline \multirow[t]{2}{*}{ GTV (cc) } & SBRT & Median (range) & $6.4(1.2-29.2)$ \\
\hline & CRT & Median (range) & $14.4(0.9-104.8)$ \\
\hline \multirow[t]{2}{*}{ PTV (cc) } & SBRT & Median (range) & $27.7(8.2-89.6)$ \\
\hline & CRT & Median (range) & $144.8(27.7-404.1)$ \\
\hline \multirow{4}{*}{\multicolumn{2}{|c|}{ Chemotherapy }} & None & 9 \\
\hline & & Neoadjuvant & 5 \\
\hline & & Concurrent & 4 \\
\hline & & Both & 3 \\
\hline
\end{tabular}

Abbreviations: ECOG Eastern Cooperative Oncology Group, FVC forced vital capacity, FEV1 forced expiratory volume in $1 \mathrm{~s}, D L c o$ carbon monoxide diffusing capacity, SBRT stereotactic body radiotherapy, CRT conventional radiotherapy, NSCLC non-small cell lung cancer, SCLC small cell lung cancer, GTV gross tumor volume, PTV planning target volume

${ }^{a}$ Two patients had two lung nodules each, and one presented with three lung nodules

${ }^{b}$ DLco information not available for four patients 
within 3 months after CRT were defined as acute toxicities.

Follow-up durations were calculated from the date that treatment was discontinued. OS was calculated using the Kaplan-Meier method from the date of treatment discontinuation until death. Clinical and dosimetric factors of patients with and without grade $\geq 3$ acute radiation pneumonitis (RP) were compared using Mann-Whitney U-tests. All statistical analyses were performed using SPSS version 21.0.

\section{Results}

\section{Patients and treatments}

A total of 21 patients were included; their demographic and clinical characteristics are shown in Table 1. Median patient age was 68 years (range, 52-88 years), and their initial or recurrent clinical stages were classified as stages IIA $(n=4)$, IIIA $(n=8)$, and IIIB $(n=9)$. All patients had forced expiratory volume in $1 \mathrm{~s}$ (FEV1) $\geq 40 \%$ of normal, making them eligible for the NPC 95-01 study [2]. Carbon monoxide diffusing capacity (DLco) was $\geq 60 \%$ in 17 patients, $\geq 40$ and $<60 \%$ in three, and $37 \%$ in one. Eighteen patients had NSCLC, and three had SCLC, with 15 receiving definitive and six receiving salvage radiotherapy.

The median SBRT dose was 54 Gy (range, 40-60 Gy), which was administered in 4 fractions (median fraction size, 14 Gy; range, 10-15 Gy/fraction). Eight patients who received 60 Gy over 4 fractions underwent treatment twice a week, whereas the others were treated daily. Seven patients were treated with SBRT using three-dimensional conformal RT (3D-CRT), whereas 13 received volumetric arc therapy. Non-isocentric technique using a Cyberknife was applied to one patient. Median time interval between the start of SBRT and the start of CRT was 8 days (range, 6-24 days). The median CRT dose was 60 Gy (range, 46-66 Gy), and the median dose per fraction was 2 Gy (range, 2-6 Gy). Only one patient received 6 Gy fractions, which is strictly defined as hypofractionated radiotherapy, rather than CRT. Nevertheless, because this study focused on the difference in fractionation regimens administered to the lung and nodal area, this patient was included. Four patients received intensity modulated $\mathrm{RT}$, and the others received $3 \mathrm{D}-\mathrm{CRT}$. Nine patients did not receive any chemotherapy due to old age ( $\geq 75$ years) and comorbidities $(n=6)$, including idiopathic interstitial pneumonia (IIP) $(n=1)$, chronic obstructive pulmonary disease (COPD) $(n=1)$, and patient refusal $(\mathrm{n}=1)$.

\section{Survival and patterns of failure}

The median follow-up duration was 12 months (range, 1-92 months). Median survival was 13 months (range, 1-92 months). The 1 year OS rate was $60.5 \%$ (Fig. 2a), whereas the 1 year LRFS and DMFS rates were 84.8 and $62.1 \%$, respectively (Fig. $2 \mathrm{~b}$ and c). The 2 year OS rate was also $60.5 \%$, whereas the 2 year LRFS and DMFS rates were 74.2 and $45.2 \%$, respectively.

Two patients experienced in-field local recurrence, combined with out-field regional and/or distant failure. The locations of the in-field recurrences were the mediastinal and low cervical nodal areas. Both patients had NSCLC, with one having recurrence after lobectomy. Neither received any chemotherapy. Five patients exhibited out-field regional failure (crude incidence rate, 24\%), with three simultaneously diagnosed with distant recurrence. All out-field regional recurrences occurred in nodal areas, not in lung fields, and all of these patients had NSCLC. Four of these patients received definitive treatment, whereas one received salvage treatment. Three did not receive any chemotherapy. The major recurrence pattern was distant failure, which occurred in nine patients (crude incidence, 43\%). Of these nine

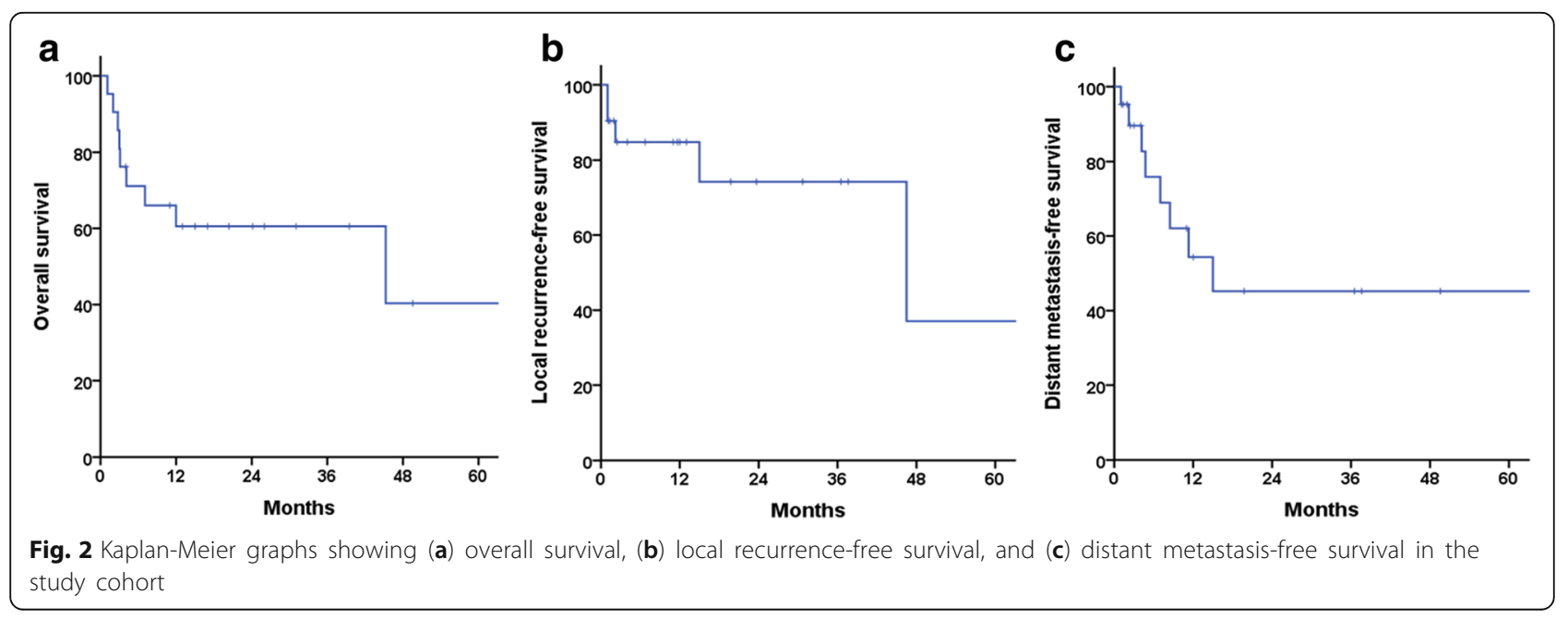


Table 2 Characteristics of three patients with grade $\geq 3$ acute radiation pneumonitis

\begin{tabular}{|c|c|c|c|c|}
\hline \multirow{2}{*}{$\frac{\text { Factors }}{\text { Radiation pneumonitis grade }}$} & & \multicolumn{3}{|l|}{ Patients } \\
\hline & & 3 & 4 & 4 \\
\hline \multicolumn{2}{|l|}{ Sex } & Male & Male & Male \\
\hline \multicolumn{2}{|l|}{ Age (year) } & 88 & 79 & 80 \\
\hline \multicolumn{2}{|l|}{ ECOG performance status } & 2 & 1 & 1 \\
\hline \multirow[t]{3}{*}{ Pre-treatment pulmonary function test } & $\mathrm{FVC}(\mathrm{L})$ & 2.5 & 2.1 & 4.8 \\
\hline & FEV1 (L) & 1.9 & 2.0 & 2.9 \\
\hline & DLCo (\%) & 85 & 37 & 84 \\
\hline \multicolumn{2}{|l|}{ Combined pulmonary disease } & None & $\| P$ & None \\
\hline \multicolumn{2}{|l|}{ Aim } & Definitive & Salvage & Salvage \\
\hline \multicolumn{2}{|l|}{ SBRT dose (Gy/fraction) } & $48 / 4$ & $40 / 4$ & $60 / 4$ \\
\hline \multicolumn{2}{|l|}{ CRT dose (Gy/fraction) } & $66 / 33$ & $60 / 10$ & $60 / 30$ \\
\hline \multirow[t]{2}{*}{ GTV (cc) } & Lung & 29.2 & 6.1 & 6.4 \\
\hline & Nodal area & 17.7 & 4.9 & 24.2 \\
\hline \multirow[t]{2}{*}{ PTV (cc) } & Lung & 89.6 & 26.5 & 30.1 \\
\hline & Nodal area & 298.2 & 27.7 & 361.0 \\
\hline \multicolumn{2}{|l|}{ Chemotherapy } & None & None & None \\
\hline \multicolumn{2}{|c|}{ Occurrence time of radiation pneumonitis (days) } & 40 & 43 & 18 \\
\hline \multicolumn{2}{|c|}{ Bilateral or unilateral lung involvement of radiation pneumonitis } & Unilateral & Bilateral & Bilateral \\
\hline \multicolumn{2}{|c|}{ Time from onset of symptoms to death (days) } & 53 & 17 & 15 \\
\hline
\end{tabular}

Abbreviations: ECOG Eastern Cooperative Oncology Group, FVC forced vital capacity, FEV1 forced expiratory volume in 1 s, DLco carbon monoxide diffusing capacity, SBRT stereotactic body radiotherapy, CRT conventional radiotherapy, GTV gross tumor volume, PTV planning target volume, IIP idiopathic interstitial pneumonia

patients, only one had SCLC, two received salvage treatment, and two did not receive chemotherapy.

\section{Toxicity}

Nine patients experienced acute radiation esophagitis, including eight with grade 2 disease and one with grade 3 disease. Except for one patient who finally progressed to grade 3 late radiation esophagitis, the other patients were successfully managed medically. Eight patients experienced acute RP, including five with grade 2 , one with grade 3 , and two with grade 4 (life-threatening) disease. The characteristics of the three patients with acute $\mathrm{RP}$ of grade $\geq 3$ are summarized in Table 2. All these patients were aged $\geq 79$ years.

One patient who initially had IIP showed poor lung function with a DLco of $37 \%$; this patient was treated with hypofractionated radiotherapy to the nodal area (60 Gy over 10 fractions). Nevertheless, as the PTV of the nodal area was very small $(27.7 \mathrm{cc})$, more conservative dose constraints were applied. Dosimetric parameters showed that his MLD was 7.1 Gy and his V20 was 9.1\% (Table 3). All other patients satisfied the institutional OAR dose constraints.

The only significant factor associated with grade $\geq 3$ $\mathrm{RP}$ was older age $(p=0.011)$. Other demographic and clinical factors, including sex, performance status, smoking history, pre-treatment pulmonary function test results, aim of radiotherapy (definitive or salvage), and combined chemotherapy, were not significantly associated with grade $\geq 3 \mathrm{RP}$. Due to the small number of patients enrolled, we could not reliably determine the dosimetric parameters, such as MLD, V5, V20, mean ipsilateral lung dose, and PTV size, predictive of grade $\geq$ 3 RP. Two patients without a history of acute RP experienced late RP of grade 2 . None of the patients experienced any grade $\geq 2$ hematologic toxicity.

\section{Discussion}

The present study describes the clinical outcomes in patients who underwent $\mathrm{S}+\mathrm{CRT}$. Definitive CCRT for unresectable or medically inoperable stage II-III NSCLC has shown local failure rates of $30-53 \%[1,2]$ and a 3 year OS rate of approximately 27\% [12]. Moreover, CCRT for LS-SCLC has shown a 2 year intrathoracic recurrence rate of $66 \%$ [5] and a 3 year OS rate of $14 \%$ [4]. The present study found that the 2 year LRFS rate was $74.2 \%$, the crude local failure rate was $24 \%$, and the 2 year OS rate was $45.2 \%$, consistent with the findings of previous studies.

Only two of our patients experienced in-field recurrences at nodal areas alone. A previous study investigated the patterns of loco-regional failure in locally advanced NSCLC patients who received definitive CCRT (66 Gy in 24 fractions) [13]. There were about a 2.5-fold 
Table 3 Dosimetric parameters for lungs in all patients

\begin{tabular}{|c|c|c|c|c|}
\hline Patients & MLD (Gy) & V5 (\%) & V20 (\%) & MILD (Gy) \\
\hline 1 & 12.8 & 53.0 & 22.7 & 18.7 \\
\hline 2 & 4.0 & 20.6 & 4.6 & 5.4 \\
\hline 3 & 15.9 & 72.2 & 27.2 & 20.8 \\
\hline $4^{\mathrm{a}}$ & 17.0 & 55.7 & 28.0 & 28.5 \\
\hline $5^{\mathrm{a}}$ & 7.1 & 42.8 & 9.1 & 7.2 \\
\hline 6 & 18.3 & 80.3 & 32.9 & 14.1 \\
\hline 7 & 18.8 & 74.0 & 37.4 & 27.0 \\
\hline $8^{\mathrm{a}}$ & 16.4 & 78.3 & 29.6 & 15.2 \\
\hline 9 & 14.9 & 47.5 & 25.9 & 20.6 \\
\hline 10 & 10.3 & 25.5 & 18.8 & 18.9 \\
\hline 11 & 13.5 & 48.5 & 22.7 & 17.1 \\
\hline 12 & 13.3 & 54.1 & 25.9 & 21.0 \\
\hline 13 & 12.1 & 60.9 & 19.0 & 17.3 \\
\hline 14 & 11.1 & 49.1 & 19.8 & 20.8 \\
\hline 15 & 13.2 & 48.1 & 20.3 & 22.1 \\
\hline 16 & 7.9 & 29.8 & 13.8 & 15.9 \\
\hline 17 & 11.6 & 50.4 & 18.9 & 17.3 \\
\hline 18 & 13.5 & 47.7 & 27.4 & 26.5 \\
\hline 19 & 14.7 & 64.3 & 23.1 & 22.0 \\
\hline 20 & 3.4 & 17.0 & 3.2 & 5.3 \\
\hline 21 & 4.9 & 26.0 & 5.3 & 8.0 \\
\hline
\end{tabular}

Abbreviations: $M L D$ mean lung dose, $V X$ volume of lung receiving at least $X \mathrm{~Gy}$, MILD mean ipsilateral lung dose

${ }^{\text {a }}$ Patients with grade $\geq 3$ acute radiation pneumonitis

higher absolute risk of primary tumor failure (16\%) than nodal failure (6\%). In the present study, we observed no recurrence in primary lung lesions which may be attributed to the administration of SBRT. The results of a randomized trial comparing SBRT and CRT in patients with inoperable stage I NSCLC (CHISEL) also support our findings, with both longer LRFS (hazard ratio $[\mathrm{HR}]=$ $0.29, p=0.002)$ and OS $(\mathrm{HR}=0.51, p=0.02)$ in the SBRT arm [10]. Although it may be worrisome that reducing PTV margins resulted in recurrences in the non-irradiated lung field between the PTVs of the primary lung tumor and LNs, there were no recurrences in any lung tissue. Because most regional recurrences occurred in nodal areas, it is important to improve nodal disease control. Although doses have been escalated to achieve greater local control, the RTOG 0617 trial, which compared regimens of 74 and 60 Gy (2 Gy/fraction), found that the higher dose regimen did not increase 2 year LRFS and OS rates [14]. This finding suggested that dose escalation in CRT may not be sufficient for improving tumor control, suggesting that SBRT may provide additional benefits.

Attempts have been made to administer SBRT to patients with locally advanced cancers. For example, a prospective, single-institution study evaluated the feasibility of CRT (59.4 Gy in 33 fractions) followed by SBRT (20 Gy in 2 fractions or 19.5 Gy in 3 fractions) in patients with stage II-III NSCLC and residual disease on positron emission tomography [15]. Over a median follow-up of 13 months, the local control rate was found to be $83 \%$, similar to our crude local control rate of $76 \%$. Additional studies are required to determine whether administration of SBRT to both lung lesions and LNs reduces the incidence of nodal in-field recurrences.

Patients administered S + CRT are likely to experience the abscopal effect of SBRT [16]. Because SBRT in patients with stage I NSCLC was performed in the absence of nodal staging and irradiation, it should result in higher rates of regional recurrence than with surgery. However, regional failure rates in patients undergoing SBRT were comparable to those in patients undergoing surgery, including LN dissection $[6,8]$. This phenomenon may be due to an abscopal effect, defined as the anti-tumor effect of radiation outside the radiotherapy field, resulting from increased tumor antigen expression and T-cell response following radiotherapy [16]. Although additional evidence is needed, the combination of CRT and SBRT may improve oncologic outcomes in patients with lung cancer through abscopal effect.

Previous studies of patients with NSCLC treated with 63-66 Gy in fractions of 1.8-2 Gy reported grade $\geq 3$ pulmonary toxicities presenting as acute complications in $4-5 \%$ of patients and late toxicities in $11 \%[1,2]$, and a prospective, single-institution study reported an incidence rate of grade $\geq 3$ acute RP of $11 \%$ [15]. Acceptable rates of severe toxicity of SBRT in patients with stage I NSCLC have been reported to range from 4.5 to $10.2 \%$ [17]. We suggest that toxicity rates may be lower with SBRT than with CRT, as SBRT has high targeting accuracy and rapid dose falloff. Moreover, the greater accuracy of SBRT resulted in smaller PTV margins. A randomized trial comparing SBRT and CRT in patients with inoperable stage I NSCLC (SPACE trial) reported a significantly lower rate of esophagitis $(8 \%$ vs. $30 \%, p=$ $0.006)$ and a lower rate of RP ( $19 \%$ vs. $34 \%, p=0.26)$ in the SBRT than in the CRT group [9]. Although the incidence rate of grade $\geq 3$ acute RP in the present study was $21 \%$, higher than in previous trials, our study population was older, with a median age of 68 years (compared with median ages of 61-63 years in previous studies), and included patients with recurrent disease after curative surgery. As age $\geq 60$ years is a predictive factor for RP [18], our patients had a greater likelihood of developing RP.

One patient in the present study with grade $4 \mathrm{RP}$ had IIP and a DLco of only 37\%. A more pronounced decrease in lung function has been reported in 
individuals with an initial DLco of $\leq 50 \%$ [19]. Lung constraints in the present study included MLD $<20$ Gy and $\mathrm{V} 20<30 \%$. A study assessing the association of dose-volume parameters with the risk of grade $\geq 3 \mathrm{RP}$ found that patients who satisfied the threshold dose-volume histogram curve, defined by V $20 \leq 25 \%$, $\mathrm{V} 25 \leq 20 \%$, V $35 \leq 15 \%$, and V $50 \leq 10 \%$, had an incidence of grade $\geq 3$ RP of only $2 \%$ [20]. Stricter lung constraints are therefore needed for older aged patients and those with poor lung function.

This study had several limitations, including its retrospective design, the small number of patients, and the heterogeneity of the enrolled patients. Although CCRT was the standard treatment, $43 \%$ of these patients did not receive any chemotherapy. Therefore, the results of the present study are not representative of standard populations. The potential numbers of candidates for S + CRT are limited, because relatively few patients present with primary lung lesions distinct from nodal areas.

\section{Conclusions}

This retrospective analysis of lung cancer patients treated with $\mathrm{S}+\mathrm{CRT}$ showed promising local control and survival rates, as well as acceptable toxicities. Stricter lung dose constraints are need for older aged patients and those with underlying pulmonary diseases. Additional studies, in larger numbers of patients and with stricter enrollment criteria, are required to confirm these findings.

\begin{abstract}
Abbreviations
3D-CRT: Three-dimensional conformal RT; AJCC: American Joint Committee on Cancer; CCRT: Concurrent chemoradiotherapy; COPD: Chronic obstructive pulmonary disease; CRT: Conventional radiotherapy; CT: Computed tomography; CXR: Chest X-rays; DLco: Carbon monoxide diffusing capacity; DMFS: Distant metastasis-free survival; FDG-PET-CT: 18-fluoro-deoxyglucose positron emission tomography; FEV1: Forced expiratory volume in $1 \mathrm{~s}$; GTV: Gross tumor volume; HR: Hazard ratio; IIP: Idiopathic interstitial pneumonia; ITV: Internal target volume; LN: Lymph node; LRFS: Local recurrence-free survival; LS-SCLC: Limited-stage small cell lung cancer; MILD: Mean ipsilateral lung dose; MLD: Mean lung dose; NSCLC: Non-small cell lung cancer; OAR: Organs at risk; OS: Overall survival; PTV: Planning target volume; RP: Radiation pneumonitis; S + CRT: Stereotactic plus conventional radiotherapy; SBRT: Stereotactic body radiotherapy; SCLC: Small cell lung cancer; V20: Volume of lung receiving at least $20 \mathrm{~Gy}$
\end{abstract}

\section{Funding}

This research did not receive any specific grant from funding agencies in the public, commercial, or not-for-profit sectors.

\section{Authors' contributions}

SSK designed the trial. SYS and EKC evaluated the clinical outcomes. YJK drafted the manuscript and was responsible for statistical considerations. All authors have read and approved the final manuscript.

\section{Ethics approval and consent to participate}

Approval to conduct this study (version 1.1 on June 2, 2017) was granted by the Institutional Review Board of Seoul Asan Medical Center (S2017-05570002), and the present retrospective study was conducted in accordance with the Helsinki Declaration.
Consent for publication

Not applicable.

\section{Competing interests}

The authors declare that they have no competing interests.

\section{Publisher's Note}

Springer Nature remains neutral with regard to jurisdictional claims in published maps and institutional affiliations.

Received: 27 March 2018 Accepted: 2 July 2018

Published online: 11 July 2018

\section{References}

1. Curran WJ Jr, Paulus R, Langer CJ, et al. Sequential vs. concurrent chemoradiation for stage III non-small cell lung cancer: randomized phase II trial RTOG 9410. J Natl Cancer Inst. 2011;103(19):1452-60. https://doi.org/ 10.1093/jnci/djr325. PubMed PMID: 21903745; PubMed Central PMCID: PMCPMC3186782. eng.

2. Fournel $P$, Robinet $G$, Thomas $P$, et al. Randomized phase III trial of sequential chemoradiotherapy compared with concurrent chemoradiotherapy in locally advanced non-small-cell lung cancer: Groupe Lyon-saint-Etienne d'Oncologie Thoracique-Groupe Francais de PneumoCancerologie NPC 95-01 study. J Clin Oncol. 2005;23(25):5910-7. https://doi. org/10.1200/jco.2005.03.070. PubMed PMID: 16087956; eng.

3. Huber RM, Flentje M, Schmidt M, et al. Simultaneous chemoradiotherapy compared with radiotherapy alone after induction chemotherapy in inoperable stage IIIA or IIIB non-small-cell lung cancer: study CTRT99/97 by the bronchial carcinoma therapy group. J Clin Oncol. 2006;24(27):4397-404.

4. Pignon J, Arriagada R. Role of thoracic radiotherapy in limited-stage small-cell lung cancer: quantitative review based on the literature versus meta-analysis based on individual data. J Clin Oncol. 1992:10(11):1819-20.

5. Warde P, Payne D. Does thoracic irradiation improve survival and local control in limited-stage small-cell carcinoma of the lung? A meta-analysis. J Clin Oncol. 1992;10(6):890-5.

6. Chang JY, Senan S, Paul MA, et al. Stereotactic ablative radiotherapy versus lobectomy for operable stage I non-small-cell lung cancer: a pooled analysis of two randomised trials. Lancet Oncol. 2015;16(6):630-7.

7. Jung I-H, Song SY, Jung J, et al. Clinical outcome of fiducial-less CyberKnife radiosurgery for stage I non-small cell lung cancer. Radiat Oncol J. 2015; 33(2):89-97

8. Robinson CG, DeWees TA, El Naqa IM, et al. Patterns of failure after stereotactic body radiation therapy or lobar resection for clinical stage I non-small-cell lung cancer. J Thorac Oncol. 2013;8(2):192-201.

9. Nyman J, Hallqvist A, Lund JA, et al. SPACE - a randomized study of SBRT vs conventional fractionated radiotherapy in medically inoperable stage I NSCLC. Radiother Oncol. 2016;121(1):1-8. https://doi.org/10.1016/j.radonc. 2016.08.015. PubMed PMID: 27600155; eng.

10. Ball D, Mai T, Vinod S, et al. MA 13.07 a randomized trial of SABR vs conventional radiotherapy for inoperable stage I non-small cell lung Cancer: TROG09. 02 (CHISEL). J Thorac Oncol. 2017;12(11):S1853.

11. Shepherd FA, Crowley J, Van Houtte P, et al. The International Association for the Study of Lung Cancer lung cancer staging project: proposals regarding the clinical staging of small cell lung cancer in the forthcoming (seventh) edition of the tumor, node, metastasis classification for lung cancer. J Thor Oncol. 2007;2(12):1067-77. https://doi.org/10.1097/JTO. 0b013e31815bdc0d. PubMed PMID: 18090577; eng.

12. Hanna N, Neubauer M, Yiannoutsos C, et al. Phase III study of cisplatin, etoposide, and concurrent chest radiation with or without consolidation docetaxel in patients with inoperable stage III non-small-cell lung cancer: the Hoosier oncology group and US. Oncology. J Clin Oncol. 2008;26(35):5755-60. https://doi.org/10.1200/jco.2008.17.7840. PubMed PMID: 19001323; eng

13. van Diessen JN, Chen C, van den Heuvel MM, et al. Differential analysis of local and regional failure in locally advanced non-small cell lung cancer patients treated with concurrent chemoradiotherapy. Radiother Oncol. 2016; 118(3):447-52. https://doi.org/10.1016/j.radonc.2016.02.008. PubMed PMID: 26900092; eng.

14. Bradley JD, Paulus R, Komaki R, et al. Standard-dose versus high-dose conformal radiotherapy with concurrent and consolidation carboplatin plus 
paclitaxel with or without cetuximab for patients with stage IIIA or IIIB nonsmall-cell lung cancer (RTOG 0617): a randomised, two-by-two factorial phase 3 study. Lancet Oncol. 2015;16(2):187-99.

15. Feddock J, Arnold SM, Shelton BJ, et al. Stereotactic body radiation therapy can be used safely to boost residual disease in locally advanced non-small cell lung cancer: a prospective study. Int J Radiat Oncol Biol Phys. 2013; 85(5):1325-31. https://doi.org/10.1016/j.jijobp.2012.11.011. PubMed PMID: 23265574; eng

16. Popp I, Grosu AL, Niedermann G, et al. Immune modulation by hypofractionated stereotactic radiation therapy: therapeutic implications. Radiother Oncol. 2016;120(2):185-94.

17. Navarro-Martin A, Aso S, Cacicedo J, et al. Phase II trial of SBRT for stage I NSCLC: survival, local control, and lung function at 36 months. J Thorac Oncol 2016 2016/07/01/;11(7):1101-1111. doi: https://doi.org/10.1016/j.jtho. 2016.03.021.

18. Claude L, Perol D, Ginestet $C$, et al. A prospective study on radiation pneumonitis following conformal radiation therapy in non-small-cell lung cancer: clinical and dosimetric factors analysis. Radiother Oncol. 2004;71(2): 175-81. https://doi.org/10.1016/j.radonc.2004.02.005. PubMed PMID: 15110451; eng

19. Lopez Guerra JL, Gomez DR, Zhuang Y, et al. Changes in pulmonary function after three-dimensional conformal radiotherapy, intensitymodulated radiotherapy, or proton beam therapy for non-small-cell lung cancer. Int J Radiat Oncol Biol Phys. 2012;83(4):e537-43. https://doi.org/10. 1016/j.jjobp.2012.01.019. PubMed PMID: 22420964; PubMed Central PMCID: PMCPMC3923580. eng

20. Jin H, Tucker SL, Liu HH, et al. Dose-volume thresholds and smoking status for the risk of treatment-related pneumonitis in inoperable non-small cell lung cancer treated with definitive radiotherapy. Radiother Oncol. 2009:91(3):427-32

\section{Ready to submit your research? Choose BMC and benefit from:}

- fast, convenient online submission

- thorough peer review by experienced researchers in your field

- rapid publication on acceptance

- support for research data, including large and complex data types

- gold Open Access which fosters wider collaboration and increased citations

- maximum visibility for your research: over $100 \mathrm{M}$ website views per year 